\section{Ärztliche Beratung via Internet}

C. Oswald

\section{Einleitung}

Nicht nur Private, der Handel, die Industrie oder zahlreiche Dienstleistungsunternehmungen wissen heute die mannigfaltigen Verwendungsmöglichkeiten des Internets zu schätzen und für ihre Interessen nutzbringend einzusetzen. Die neue Technologie macht auch vor der Ärzteschaft nicht halt. Die medizinische Online-Beratung - aber auch der Medikamentenhandel - übers Internet stösst auf grosse Nachfrage.

Als im Herbst 1998 der russische Regattasegler Wiktor Jasikow auf hoher See durch einen Abszess in Lebensgefahr geriet, sandte er per Satellit einen Notruf ins Internet. Damit rückte die Telemedizin auf einen Schlag in den Fokus des öffentlichen Interesses, dies umso mehr, als der Vorfall ein gutes Ende nahm: Ein Bostoner Arzt erklärte Jasikow per E-mail die notwendigen Behandlungsschritte. Der Solosegler operierte sich selbst und rettete sich dadurch das Leben [1].

Unter weniger spektakulären Umständen, doch oft mit der gleichen verzweifelten Hoffnung, suchen Patienten ${ }^{1}$ auch in der Schweiz medizinische Hilfe im Internet. Diese finden sie - um lediglich ein Beispiel unter vielen zu nennen - via Website des Universitätsspitals Zürich. Dort betreut eine Ärztin in Zusammenarbeit mit Spezialistinnen und Spezialisten des Spitals eine E-mail-Box und beantwortet in der Regel Anfragen innerhalb von 48 Stunden [2].

Eine gewisse Ablehnung der Mediziner gegenüber dem Internet ist nicht unbegründet. Während es ausgezeichnete Quellen medizinischer Information und Beratung gibt, versuchen gleichzeitig weltweit Tausende von Scharlatanen, auf unlautere Weise Profit zu schlagen, im Wissen darum, dass in medizinischen Belangen unerfahrene oder verzweifelte Patienten nicht zwischen fundierter Analyse und Quacksalberei unterscheiden können.

1 Wenn im nachfolgenden vom "Arzt» oder vom «Patient» die Rede ist, ist gleichsam auch die "Ärztin" oder die "Patientin" gemeint. Die Vereinfachung dient der besseren Lesbarkeit.

2 Ein Überblick über schweizerische, deutsche und englischsprachige Internetberatung findet sich z.B. unter [3].

Korrespondenz:

Dr. Christoph Oswald

Haldenstrasse 167

CH-8055 Zürich
Die Online-Beratung ist bereits heute ein Faktum und wird von Ärzten und Patienten rege benutzt ${ }^{2}$. Immer mehr Medizinal- und Gesundheitsportale öffnen ihre Pforten. Die ersten medizinischen Callcenters sind Tatsache geworden [4]. Oft werden hingegen die damit verbundenen Probleme und Fragestellungen erst im nachhinein erkannt.

Das Spital, die Klinik, die Ärztegemeinschaft, die Gruppenpraxis oder der einzelne praktizierende Arzt wird sich möglicherweise vor dem "Internetauftritt" folgende Fragen stellen: Vereinbarkeit der Internetberatung mit den Standesregeln? Zulässigkeit der Beratung von ausserkantonalen oder im Ausland wohnenden Patienten? Haftung für allfällige Fehldiagnosen oder Fehlbehandlungen? Im Zusammenhang mit der Haftungsfrage wird im internationalen Verhältnis auch die Frage nach dem Gerichtsstand, dem anwendbaren Recht und der Anerkennung bzw. Vollstreckung eines im Ausland ergangenen Urteils hier in der Schweiz relevant. Auf diese Fragen versucht der vorliegende Artikel eine Antwort zu geben. Nicht beleuchtet werden hingegen die Themenkreise Werbung, Rechnungsstellung für erfolgte OnlineAuskünfte, Datenschutz im Internet und Medikamentenverkauf via Internet.

An dieser Stelle kann auf den bereits im Jahre 1999 in der Schweizerischen Ärztezeitung erschienenen Artikel zum Thema "Telemedizin und Standesrecht" verwiesen werden. Die Autoren Hans Werner Iff und Hanspeter Kuhn geben dort einen wertvollen Überblick über sich im Zusammenhang mit der Telemedizin stellende Rechtsfragen [5].

\section{Zulässigkeit der Internetberatung}

\section{Grundsätzliches}

Die Standesordnung FMH vom 12. Dezember 1996 umschreibt in Art. 3 den Grundsatz der ärztlichen Berufsausübung: "Arzt und Ärztin üben ihren Beruf sorgfältig und gewissenhaft aus und erweisen sich dadurch des Vertrauens der Ratsuchenden und der Öffentlichkeit würdig. Voraussetzung dafür sind persönliche Integrität und berufliche Kompetenz.»

Wie der Arzt den Behandlungsauftrag zu erfüllen hat, wird in Art. 7 der Standesordnung näher statuiert: "Arzt und Ärztin haben die persönliche Beziehung zum Patienten oder zur Patientin soweit als möglich zu gewährleisten. Sie sorgen für eine persönliche Betreuung ihrer Patienten und Patientinnen, in dem Umfang, wie es deren Krankheitszustand erfordert. [...] Die regelmässige Behandlung allein aufgrund schriftlich, telefonisch oder elektronisch übermittelter Auskünfte oder Berichte von Drittpersonen ist mit einer gewissenhaften Berufsausübung unvereinbar." [6]

Vor allem aufgrund der letztgenannten Bestimmung, welche die regelmässige - und wohl ausschliessliche - Fernbehandlung unterbinden möchte, drängt sich die Frage auf nach der Zulässigkeit der Ferndiagnose oder der Fernbehandlung im Einzelfall. 
Nach wohl richtiger Auffassung kann und soll es nicht Aufgabe des Standesrechts sein, im einzelnen vorzuschreiben, wann eine telefonische Beratung oder die Beantwortung einer Patientenanfrage per Internet (E-mail) zulässig ist und in welchen Fällen nicht [5]. Der gewissenhafte Arzt wird im Einzelfall prüfen, ob die ihm gelieferten Daten ausreichend sind oder ob er weitere Angaben des Patienten benötigt, um einen verlässlichen Tip abgeben oder eine Ferndiagnose stellen zu können. Er wird entscheiden müssen, ob es unabdingbar ist, den Patienten zu sehen oder nicht. Ferner wird er sich auch darüber Rechenschaft ablegen, ob er für die Beantwortung der Frage überhaupt genügend fachlich qualifiziert ist oder ob er dem Patienten nicht vielmehr empfehlen muss, seine Fragen einem Spezialisten vorzulegen oder bei einem solchen vorstellig zu werden. All dies sind Entscheidungen, die der Arzt unter dem Gesichtspunkt der Behandlungsqualität zu treffen hat. Dieselben Entscheidungen hat der Arzt aber auch bei bereits bestehendem Arzt-Patienten-Verhältnis immer wieder von neuem zu treffen.

Auch wenn wohl in der überwiegenden Zahl der Fälle die sorgfältige und gewissenhafte Berufsausübung eine persönliche Kontaktnahme mit dem $\mathrm{Pa}$ tienten erforderlich macht, so ist im Einzelfall eine Fernberatung nicht a priori ausgeschlossen. Denn angesichts des in der Präambel der Standesordnung festgeschriebenen Grundsatzes, wonach das gesundheitliche Wohl des Menschen oberstes Ziel ärztlichen Handelns ist, lässt es sich bei gegebenen Voraussetzungen mit einer gewissenhaften Berufsausübung sehr wohl vereinbaren, dem Patienten die Mühen eines Arztbesuches - möglicherweise auch bloss einstweilen - zu ersparen. Zu denken ist nicht zuletzt an gehbehinderte oder weit abgelegene Patienten, für die das Aufsuchen einer Arztpraxis nur mit grossem Aufwand oder gar Schmerzen möglich wäre.

Beratung von Ausserkantonalen und Ausländern Zwar gestattet das Bundesgesetz betreffend die Freizügigkeit des Medizinalpersonals in der Schweizerischen Eidgenossenschaft vom 19. Dezember 1877 denjenigen Ärzten, die nach Massgabe dieses Gesetzes ein eidgenössisches Diplom erworben haben, die freie Ausübung ihres Berufes im Gebiete der ganzen Eidgenossenschaft (Art. 1) [7]. Denselben freien $\mathrm{Zu}$ gang zum Markt wird den Ärzten auch durch das Bundesgesetz über den Binnenmarkt (Binnenmarktgesetz) vom 6. Oktober 1995 in Art. 1 und 2 zugesichert [8].

Hingegen können die Kantone die selbständige Ausübung der ärztlichen Tätigkeit einer Bewilligungspflicht unterstellen. So sieht beispielsweise das Gesundheitsgesetz des Kantons Zürich vom 4. November 1962 [9] in \$16 vor, dass ausserkantonale Ärzte nur dann keiner Bewilligung zur selbständigen ärztlichen Tätigkeit bedürfen, wenn es sich um im Grenzgebiet eines benachbarten Kantons niedergelassene und dort praxisberechtigte, eidgenössisch diplomierte Ärzte handelt, die ihre Berufstätigkeit von ihrem Wohnort aus in den Grenzgebieten des Kantons
Zürich ausüben oder sofern sie in besonderen Einzelfällen vom behandelnden Arzt oder vom Kranken zugezogen werden.

Dasselbe gilt grundsätzlich auch im internationalen Verhältnis. Auch nach Inkrafttreten der bilateralen Abkommen Schweiz-EU wird die Registrierungspflicht ausländischer Ärzte in gewissen Staaten nicht wegfallen.

In Anbetracht der vorgenannten Bestimmungen muss man sich mit guten Gründen fragen, ob die via Internet erfolgende Beratungstätigkeit eines Arztes, der eine ausserkantonale oder eine ausländische Anfrage beantwortet, als ausserkantonale Berufsausübung bzw. als eine Berufsausübung im Ausland zu qualifizieren ist und entsprechend gegen die Bewilligungs- oder Registrierungspflicht verstösst.

Nach Ansicht des Autors ist dies nicht der Fall. Eröffnet ein Arzt mit Praxis hier in der Schweiz eine Website, stellt dort ein elektronisches Frageformular zur Verfügung oder gibt seine E-mail-Adresse bekannt und erteilt einem in einem anderen Kanton oder im Ausland lebenden Patienten auf Anfrage medizinische Auskünfte und Ratschläge, so wäre es lebensfremd anzunehmen, der Arzt sei dadurch ausserkantonal oder im Ausland tätig geworden. Das Tätigkeitsgebiet bzw. der Handlungsort ist und bleibt nach wie vor der Ort, wo der Arzt seine Praxis führt. Dort konsultiert er seine medizinische Literatur, dort tätigt er seine Abklärungen und dort stellt er seine Überlegungen an, die schliesslich zur Beantwortung der Anfrage des Patienten führen. Dass er das Resultat seiner Diagnose oder die Therapievorschläge via E-mail dem Patienten im Ausland zukommen lässt, verlagert den Behandlungsort nicht ins Ausland. Oder anders formuliert: Der Patient aus dem Ausland liefert dem Arzt auf elektronischem Weg die Daten, anhand dieser hier in der Schweiz seine Beurteilung und somit seine eigentliche Hauptleistung vornimmt. Die Rücksendung der Anwort via E-mail an den Patienten stellt "nur" eine Nebenleistung des Arztes dar, insofern als vereinbart wurde, dass der Arzt seine Ergebnisse auf diesem Weg bekanntgibt. Ebenso gut hätte vereinbart werden können, der Patient soll sich nach Ablauf einer gewissen Zeit beim Arzt telefonisch erkundigen. Im letzteren Fall käme niemand auf die Idee, einen ausserkantonalen oder ausländischen Handlungsort des Arztes anzunehmen.

Von der Frage des Ortes der Berufsausübung zu trennen ist die Frage, ob der Arzt durch den Internetauftritt im Ausland Werbung macht und damit den einzelnen nationalen Werbevorschriften, Lauterkeitsrechts- oder Verbraucherschutzrechtsbestimmungen untersteht (vgl. dazu auch die Ausführungen unter "Verbrauchervertrag [Konsumentenvertrag]»). In diesem Zusammenhang kann darauf hingewiesen werden, dass das Europäische Parlament "Richtlinien über den elektronischen Geschäftsverkehr" gebilligt und am 17. Juli 2000 veröffentlicht hat [10]. Die Mitgliedstaaten haben diese Richtlinien bis zum 17. Januar 2002 in nationales Recht umzusetzen. Gemäss Art. 3 der Richtlinie wird für die rechtlichen Rahmenbedingungen eines Anbieters von Dienstleistun- 
gen oder Produkten das allgemeine europarechtliche Herkunftslandprinzip für Internetdienste eingeführt werden, d.h. Dienstanbieter unterliegen bei grenzüberschreitender Kommunikation den Regeln des Sitzlandes. So hat ein Dienstanbieter, der seine Dienste entsprechend den Vorgaben seines Heimatrechts erbringt, keine zusätzlichen Restriktionen im Abrufstaat zu befürchten. Dies hat grundsätzlich auch für die Anbieter von medizinischen Dienstleistungen oder medizinischen Produkten Gültigkeit. Wieweit jedoch die einzelnen Mitgliedstaaten von dem in Art. 3 Abs. 4 der Richtlinie vorbehaltenen Schutzklauselverfahren Gebrauch machen und zum Schutz der öffentlichen Gesundheit und Sicherheit sowie zum Schutz der Verbraucher Ausnahmeregelungen treffen, die das Herkunftslandprinzip ausser Kraft setzen könnten, bleibt abzuwarten (vgl. [11]).

\section{Haftungsprobleme nach schweizerischem Recht}

Möglicherweise erweist sich nach erfolgter Beratung eines Patienten via Internet, dass die vom Arzt gestellte Diagnose oder die verordnete Therapie falsch bzw. ungeeignet war und dem Patienten dadurch ein Schaden erwachsen ist. Damit stellt sich das Problem der Haftung des Arztes für seine unsachgemässe Beurteilung, d.h. für allfällig erfolglose oder gar schadenverursachende Therapievorschläge.

Das Bundesgericht [12] hatte sich im Jahre 1990 mit einer telefonischen Auskunft eines Arztes bzw. dessen Arztgehilfin an die Mutter eines Kleinkindes zu befassen. Der am Telefon erteilte Rat stellte sich in der Folge als falsch heraus und war mitursächlich für die kurz darauf eingetretene Schädigung des Kleinkindes. Im Urteil wird dazu folgendes ausgeführt:

"An eine Telefondiagnose und eine Telefontherapie aber sind grundsätzlich dieselben Anforderungen zu stellen wie an die ärztliche Sorgfaltspflicht bei persönlicher Kontaktnahme [...]. Wird einem Arzt telefonisch ein Krankheitsbild vorgetragen, hat er bei objektiv gegebenem Verdacht auf eine bestimmte Krankheit nach angenommenem Auftrag nötigenfalls die gebotenen Untersuchungen durchzuführen und bei Unvermögen des Patienten, die Praxis aufzusuchen, einen Hausbesuch vorzunehmen oder andere geeignete Massnahmen (Spitaleinweisung usw.) zu veranlassen [...]. Mindestens aber ist er zur Aufklärung über die möglichen Risiken des ihm vorgetragenen Krankheitsbildes verpflichtet. Dieser Pflicht ist der Beklagte nicht nachgekommen.» ${ }^{3}$

3 Vgl. [12] und [5], wo der Sachverhalt ausführlicher zusammengefasst wird.

4 Vgl. auch [16]: "Als angenommen gilt ein nicht sofort abgelehnter Auftrag, wenn er sich auf die Besorgung solcher Geschäfte bezieht, die der Beauftragte kraft obrigkeitlicher Bestellung oder gewerbsmässig betreibt oder zu deren Besorgung er sich öffentlich empfohlen hat."
Diese Ausführungen des Bundesgerichts zur Telefondiagnose dürften für die Gerichte auch bezüglich Beratung via Internet zukünftig wegweisend sein, handelt es sich doch sowohl bei telefonischen Auskünften als auch bei der Online-Beratung um Fernberatungen, deren Qualität mit dem gleichen Massstab zu messen ist.

\section{Haftung des Arztes nach Vertragsrecht}

Heute besteht Einigkeit darüber, dass das Vertragsverhältnis zwischen Arzt und Patient einen Auftrag im Sinne von Art. 394 ff. OR darstellt [13]. Demgemäss haftet der Beauftragte (Arzt) gegenüber dem Auftraggeber (Patient) für getreue und sorgfältige Ausführung des ihm übertragenen Geschäftes (Art. 398 Abs. 2 OR).

Die Anforderungen an die Sorgfaltspflicht, die einem Arzt zuzumuten ist, lassen sich nicht ein für allemal festlegen. Sie richten sich vielmehr nach den Umständen des Einzelfalles, namentlich nach der Art des Eingriffs oder der Behandlung, den damit verbundenen Risiken, dem Ermessensspielraum, den Mitteln und der Zeit, die dem Arzt im einzelnen Fall zur Verfügung stehen, sowie nach dessen Ausbildung und Leistungsfähigkeit. Überholt ist die Ansicht, wonach die Arzthaftung auf grobe Verstösse gegen die Sorgfaltspflicht zu beschränken sei (vgl. [14]).

Fragen kann man sich, ob die Online-Beratung eines Patienten überhaupt eine vertragliche Beziehung zwischen Arzt und Patient entstehen lässt oder ob es sich lediglich um eine sogenannte Gefälligkeitshandlung (ohne vertragliche Bindung) handelt. $\mathrm{Zu}$ denken ist insbesondere an jene Fälle, bei denen die Beratung kostenlos erfolgt.

Vorauszuschicken ist, dass für die Bejahung eines Auftragsverhältnisses, mithin eines Vertrages, die Vereinbarung einer Vergütung nicht Bedingung ist. Art. 394 Abs. 3 OR hält fest, dass eine Vergütung $\mathrm{zu}$ leisten ist, "wenn sie verabredet oder üblich ist". Das Gesetz lässt somit auch Raum für unentgeltliche Auftragsverhältnisse.

Preist ein Arzt seine Online-Beratungsdienste im Internet an, so darf aufgrund dieses Umstandes geschlossen werden, dass sich der Arzt als Fachmann auf medizinischem Gebiet bei Beantwortung von Anfragen vertraglich binden und sich bei schuldhafter Verletzung der Vertragspflichten auch den entsprechenden Rechtsfolgen unterziehen werde. Die Anfrage des Patienten stellt rechtlich den Antrag zum Vertragsabschluss dar. Die Rückmeldung des Arztes, er habe die Anfrage erhalten und werde diese beantworten, oder die direkte Beantwortung der Anfrage durch den Arzt ist als entsprechende Annahme auszulegen, wodurch das Vertragsverhältnis zwischen Arzt und Patient zustande kommt. Nicht ratsam ist es, auf eine Anfrage eines Patienten nicht zu reagieren. Denn das Gesetz kennt auch die stillschweigende Annahme, falls eine ausdrückliche Annahme bei der besonderen Natur eines Geschäftes oder nach den Umständen nicht zu erwarten ist [15]. ${ }^{4}$ Möchte oder kann der Arzt die Anfrage nicht beantworten, so tut er gut daran, der anfragenden Person umgehend mitzutei- 
len, er werde oder könne ihre Frage überhaupt nicht oder erst nach einer bestimmten Zeit, z.B. nach seiner Ferienrückkehr, beantworten. Empfehlenswert ist auch, bereits auf der Website, wo die Beratung via Internet angepriesen wird, die Hinweise anzubringen, wonach (vgl. dazu auch [5]):

- es dem Arzt freigestellt ist zu entscheiden, ob er die Anfrage beantwortet;

- es dem Arzt insbesondere freigestellt bleibt, Anfragen aus dem Ausland zu beantworten $;^{5}$

- ein Vertrag erst mit ausdrücklicher Annahme (Rückmeldung, Antwort) in Kraft treten soll;

- Anfragen in der Regel innert einer bestimmten Frist (z.B. innert Wochenfrist) beantwortet werden, dafür jedoch keine Gewähr abgegeben werden kann.

Die Beratungstätigkeit des Arztes im Internet untersteht somit primär den Regeln der Vertragshaftung. (Auf die Frage, wieweit eine Vertragsverletzung zugleich eine deliktische Handlung darstellt und die Rechtsfolgen der ausservertraglichen Haftung gemäss Art. 41 ff. OR auslöst, soll an dieser Stelle nicht näher eingegangen werden.)

Auch das Bundesgericht geht nur dann von einer (vertragslosen) Gefälligkeitshandlung aus, die immerhin bei leichtfertig unrichtigen Angaben die ausservertragliche Haftung gemäss Art. 41 ff. OR nach sich ziehen kann, wenn die Gefälligkeit weder in Ausübung eines Gewerbes noch gegen Entgelt erfolgt (vgl. [17]). Es ist evident, dass die erste negative Voraussetzung (keine Ausübung eines Gewerbes) bei der ärztlichen Beratungstätigkeit nicht gegeben ist.

Vor dem Hintergrund der vorstehenden Ausführungen ist deshalb davon auszugehen, dass durch die Beratung via Internet regelmässig ein Vertragsverhältnis zwischen Arzt und Patient begründet wird und der Arzt deshalb bei schuldhafter Verletzung seiner Vertragspflichten, mithin bei unsachgemässer Behandlung dem Patienten gegenüber schadenersatzpflichtig wird.

Wenn sich ein Patient von verschiedenen Ärzten behandeln lässt, die überdies in verschiedenen Kantonen praktizieren, und in der Folge der Patient durch die Behandlung geschädigt wird, so kann sich für den Patienten u.a. die Frage stellen, ob er jeden Arzt einzeln im betreffenden Kanton einklagen muss, oder ob ihm ein einheitlicher Gerichtsstand zur Verfügung steht. Das am 1. Januar 2001 in Kraft getretene Bundesgesetz über den Gerichtsstand in Zivilsachen (GestG) bringt diesbezüglich eine gewisse Vereinfachung [18]: Gemäss Art. 7 Abs. 1 GestG ist bei Vorhandensein mehrerer Streitgenossen das für eine beklagte Partei zuständige Gericht auch für die andern beklagten Parteien zuständig. ${ }^{6}$

5 Vgl. nachstehend «Haftungsprobleme im internationalen Verhältnis".

6 Insofern bedürfen die Ausführungen bei [5] unter Ziff. 3 bei [16] einer Ergänzung.
Rechtsfolgen der unentgeltlichen Beratung

Wenn nach der hier vertretenen Meinung die allfällige Unentgeltlichkeit der Online-Beratung zwar keinen Einfluss hat auf die Qualifizierung des ArztPatienten-Verhältnisses als Vertrag (Auftrag), so kann sich die Unentgeltlichkeit jedoch auf das Mass der Haftung auswirken.

Art. 99 Abs. 2 OR bestimmt, dass sich das Mass der Haftung nach der besonderen Natur des Geschäftes richtet und insbesondere milder beurteilt wird, wenn das Geschäft für den Schuldner (Arzt) keinerlei Vorteil bezweckt. Auch Art. 43 OR, der aufgrund der Verweisung von Art. 99 Abs. 3 OR im Vertragsrecht Anwendung findet, sieht vor, dass der Richter bei der Bestimmung von Art und Grösse des Ersatzes für den eingetretenen Schaden nebst der Grösse des Verschuldens auch die Umstände zu würdigen hat.

Angesichts dieser gesetzlichen Bestimmungen vertritt die überwiegende Lehrmeinung die Ansicht, dass bei einem unentgeltlichen Auftrag oder bei einer Gefälligkeitshandlung die Unentgeltlichkeit aus Billigkeitserwägungen bei der Bemessung des Schadenersatzes zu berücksichtigen ist [19]. Allerdings ist an dieser Stelle zu bemerken, dass eine unentgeltliche Beratung von Patienten wohl kaum aus rein altruistischen Motiven erfolgt, sondern sich hinter der Unentgeltlichkeit wiederum handfeste wirtschaftliche Überlegungen verbergen ...

$\mathrm{Ob}$ und wieweit unter diesen Umständen die Gerichte die vordergründige Unentgeltlichkeit als Schadenersatzermässigungsgrund berücksichtigen würden, muss einstweilen offengelassen werden.

\section{Haftungsprobleme im internationalen Verhältnis}

\section{Übersicht}

Während im Binnenverhältnis, d.h. bei einem in der Schweiz lebenden Patienten und einem in der Schweiz praktizierenden Arzt, Klagen eines Patienten gegen den Arzt wegen Vertragsverletzung beim zuständigen Gericht am Wohnsitz des Arztes oder am Ort der Praxistätigkeit einzuleiten sind und regelmässig schweizerisches Recht zur Anwendung kommt, so ist die Rechtslage im internationalen Verhältnis (Schweizer Arzt, im Ausland wohnender Patient) selbst für Juristen verworren. Lässt ein Arzt beispielsweise einem in Mexiko wohnenden Peruaner via E-mail eine Beratung oder eine Auskunft zukommen, die sich im nachhinein als falsch und schadenverursachend herausstellt, so ist kaum abschätzbar, vor welchen Gerichten sich der Schweizer Arzt zu verantworten hätte und welches Recht zur Anwendung gelangen würde. Denkbar ist, dass sich ein Gericht in Mexiko mit der Angelegenheit befassen und dabei mexikanisches oder peruanisches Recht anwenden würde. Dem Arzt, der sich nicht auf das Verfahren vor dem ausländischen Gericht einlassen würde und gegen den ein Urteil erginge, das ihn zu Schadenersatz verpflichtet, bliebe einzig noch die Hoffnung, dass die Schweiz das im Ausland ergangene Urteil nicht anerkennen und vollstrecken werde. 
Die nachstehenden Ausführungen sind zwangsläufig eher juristischer Natur. Sie verfolgen nicht den Zweck, den Arzt zu verunsichern, sondern sie wollen ihm aufzeigen, dass er sich bei einer allfälligen Beratung von im Ausland wohnenden Patienten unter Umständen mit Problemstellungen konfrontiert sehen kann, auf die nicht einmal die Juristen eine klare Antwort zu geben vermögen.

Gerichtsstand, anwendbares Recht, Anerkennung und Vollstreckung

\section{Rechtslage gemäss Bundesgesetz über das Internationale Privatrecht (IPRG)}

Die Anwort auf die Frage, ob ein im Ausland ergangenes Urteil hier in der Schweiz anerkannt und vollstreckt wird, kann im Bundesgesetz über das Internationale Privatrecht vom 18. Dezember 1987 (IPRG) [20] gesucht werden. Dieses Gesetz regelt im internationalen Verhältnis nicht nur die Zuständigkeit der schweizerischen Gerichte oder Behörden und das im Falle der Zuständigkeit anzuwendende Recht, sondern auch die Voraussetzungen der Anerkennung und Vollstreckung ausländischer Entscheidungen (Art. 1 IPRG).

So sieht beispielsweise Art. 149 Abs. 2 lit. b IPRG vor, dass eine ausländische Entscheidung über obligationenrechtliche Ansprüche in der Schweiz anerkannt wird, wenn sie Ansprüche aus Verträgen mit Konsumenten betrifft und am Wohnsitz oder am gewöhnlichen Aufenthalt des Konsumenten ergangen ist und dabei gewisse weitere Voraussetzungen erfüllt sind. ${ }^{7}$ Art. 120 IPRG bestimmt überdies, dass Konsumentenverträge dem Recht desjenigen Staates unterstehen, in dem der Konsument seinen gewöhnlichen Aufenthalt hat und eine Rechtswahl hinsichtlich des auf den Vertrag anzuwendenden Rechts ausgeschlossen ist.

Nicht auszuschliessen ist daher, dass die schweizerischen Behörden gestützt auf das IPRG ein in Mexiko ergangenes Urteil, das den Arzt zu Schadenersatz verpflichtet, hier in der Schweiz anerkennen und vollstrecken würden.

\section{Rechtslage gemäss Lugano-Übereinkommen (LugÜ)}

Allgemeines: Bezüglich der Fragen des Gerichtsstandes sowie der Anerkennung eines ausländischen Urteils findet das IPRG allerdings dann keine Anwendung, wenn als Vertragsparteien Staatsangehörige derjenigen Staaten beteiligt sind, die dem Übereinkommen über die gerichtliche Zuständigkeit und die Vollstreckung gerichtlicher Entscheidungen in Zivil- und Handelssachen, abgeschlossen in Lugano am 16. September 1988 (Lugano-Übereinkommen) [22], beigetreten sind. Die Schweiz sowie die Mehrzahl der europäischen Staaten haben sich diesem Übereinkommen angeschlossen. ${ }^{8}$

Der Arzt, der mit einer eigenen Website im Internet auftritt und seine Beratungsdienste anpreist, wird wohl zur Hauptsache mit Anfragen aus dem deutschsprachigen und aus dem übrigen europäischen Raum und wohl eher selten mit Fragen aus Übersee kon- frontiert werden. Im folgenden soll daher kurz die Regelung gemäss Lugano-Übereinkommen beleuchtet werden.

Im Lugano-Übereinkommen finden sich Gerichtsstandsbestimmungen sowie Anerkennungs- und Vollstreckungsbestimmungen. Nicht geregelt ist hingegen die Frage des auf einen Vertrag anwendbaren Rechts. Diese Frage wird vom jeweiligen Gericht, das sich für die Beurteilung der Sache zuständig erklärt, quasi in eigener Regie, d.h. gestützt auf das entsprechende Landesrecht, beurteilt. Insbesondere hat das Gericht auch zu entscheiden, ob es eine allfällige Rechtswahl der Parteien für verbindlich erachtet oder nicht.

Verbrauchervertrag (Konsumentenvertrag): Wie das IPRG (Art. 120) sieht auch das Lugano-Übereinkommen in Art. 14 Abs. 1 bezüglich Verbraucherverträge vor, dass der Verbraucher (Patient) die Möglichkeit hat, die Klage gegen den Vertragspartner (Arzt) vor den Gerichten des Staates zu erheben, in dessen Hoheitsgebiet der Verbraucher seinen Wohnsitz hat.

Damit jedoch die spezielle Gerichtsstandsbestimmung von Art. 14 Abs. 1 LugÜ zur Anwendung gelangt und der Konsument bzw. Patient in seinem Heimatstaat Klage erheben kann, muss bei Dienstleistungsverträgen dem Vertragsabschluss im Staat des Wohnsitzes des Verbrauchers (Patient) ein ausdrückliches Angebot oder eine Werbung vorausgegangen sein, und der Verbraucher muss in diesem Staat die zum Abschluss des Vertrags erforderlichen Rechtshandlungen vorgenommen haben (Art. 13 Ziff. 3 lit. a und b LugÜ). ${ }^{9}$

Es stellt sich nun die Frage, ob das auf der Website des Arztes zu findende Angebot, auf Anfrage medizinische Beratung via E-mail zu erhalten, als Werbung im Sinne von Art. 13 Ziff. 3 LugÜ zu qualifizieren ist. Wie in so vielen anderen Fragen gehen auch hier die Meinungen auseinander: Während einzelne Autoren diese Frage generell bejahen, ${ }^{10}$ geben andere eine differenziertere Antwort und weisen m.E. zu Recht darauf hin, dass ein Anbieter einen spezifischen Bezug zum Markt des Konsumentenstaates her-

7 Heute wird die Ansicht vertreten, dass Arzt-Patienten-Verträge als Konsumentenverträge zu qualifizieren sind und somit speziellen Rechtsfolgeregelungen unterstehen. Vgl. [21].

8 Vertragsstaaten sind zurzeit: Belgien, Dänemark, Deutschland, Finnland, Frankreich, Griechenland, Irland, Island, Italien, Luxemburg, Niederlande, Norwegen, Österreich, Portugal, Schweden, Schweiz, Spanien, Vereinigtes Königreich.

9 Eine analoge Bestimmung kennt auch das IPRG: vgl. [23].

10 Vgl. z.B. [24]: «Voraussetzung ist aber u.a., dass der Anbieter zuvor in jenem Staat geworben oder Angebote gemacht hat und der Konsument in diesem Staat die zum Vertragsabschluss erforderlichen Rechtshandlungen vorgenommen hat [...]. Diese beiden Voraussetzungen dürften für Angebote via Internet meistens erfüllt sein. Tätigt ein Konsument via Internet ein Geschäft, so wird er zuvor immer ein Angebot oder eine Werbung auf seinem Bildschirm zu sehen bekommen. Auch die für den Vertragsabschluss nötige Handlung wird er in der Regel an seinem Computer vornehmen. Es kommt folglich nur noch darauf an, dass der Konsument einen Computer an seinem gewöhnlichen Aufenthaltsort benutzt hat und es gilt das dortige Recht." 
stellen muss, damit die Anwendung der konsumentenschutzrechtlichen Schutzbestimmungen gerechtfertigt ist: "Allein die Tatsache, dass eine Website eines Anbieters technisch gesehen weltweit abgerufen werden kann, bedeutet also nicht, dass ein Unternehmer weltweit geworben hat. Vielmehr sind der Inhalt der Website sowie allfällige weitere Umstände zu berücksichtigen.» [25] ${ }^{11}$ Der blosse Hinweis auf einer Website auf die Möglichkeit, Fragen an den Arzt $\mathrm{zu}$ stellen und von diesem beraten zu werden, stellt nach richtiger Lesart keine Werbung im Sinne der Verbraucherschutznormen dar und begründet demnach auch keinen Gerichtsstand im Wohnsitzstaat des Konsumenten.

Zudem ist ungeklärt, ob bereits die Tatsache, dass die Beratung unentgeltlich erfolgt, der Annahme eines Konsumvertrags entgegensteht (so [27]).

Verneint man das Vorliegen einer Verbrauchersache, so entfällt auch der besondere Gerichtsstand am ausländischen Wohnsitz des Patienten. Der Gerichtsstand würde sich demnach nach der für die übrigen Verträge geltenden Bestimmung von Art. 5 Ziff. 1 LugÜ richten, wonach Ansprüche aus einem Vertrag vor dem Gericht des Ortes, an dem die Verpflichtung erfüllt worden ist oder zu erfüllen gewesen wäre, eingeklagt werden können. Da nach der hier vertretenen Ansicht der Ort der Berufsausübung und der Erfüllungsort sich am Ort der Praxis des Arztes befinden (vgl. vorne "Beratung von Ausserkantonalen und Ausländern»), wäre in diesem Fall ein inländisches Gericht zuständig. Anzumerken bleibt, dass diese Gerichtsstandsbestimmung nur für jene Staaten Geltung hat, die dem Lugano-Übereinkommen beigetreten sind (vgl. Fussnote 7). Ob ein Inselstaat in der Südsee eine analoge Gerichtsstandsbestimmung kennt und den Insulaner im Ergebnis zur Erhebung der Klage in die Schweiz verweist oder aber sich selbst für die Beurteilung der Streitsache als zuständig erklärt, entzieht sich der Kenntnis des Autors.

Haftung aus Delikt: Selbst wenn man sich der m.E. zutreffenden Meinung anschliesst, dass nicht jeder Internetauftritt eines Arztes zugleich als Werbung im Ausland betrachtet werden kann und demzufolge der besondere vom LugÜ für Konsumenten- bzw. Verbraucherstreitigkeiten zur Verfügung gestellte Wohnsitzgerichtsstand des Patienten nicht zur Anwendung gelangt, ist die Gefahr nicht gebannt, dass ein im Ausland wohnender Patient den Schweizer Arzt nicht doch im Ausland einklagt und das ausländische Gericht zu Recht auf die Klage eintritt.

Denkbar ist nämlich, dass der geschädigte Patient den Arzt nicht aufgrund einer Vertragsverletzung zu belangen versucht, sondern als Haftungsgrund ein sogenannt deliktisches Verhalten des Arztes geltend macht, mithin seine Klage auf die Bestimmungen über die ausservertragliche Haftung stützt. (Eine Vertragsverletzung kann auch in der Schweiz zugleich

11 Vgl. auch [26]: «Wird Werbung irgendwo auf dem Information Highway plaziert und von einem Benutzer in einem anderen Staat abgerufen, so dürfte dieses Erfordernis nicht erfüllt sein.» deliktischer Natur sein, sofern die entsprechenden Voraussetzungen gemäss den Bestimmungen über die unerlaubte Handlung, Art. 41 ff. OR, erfüllt sind.)

Während das IPRG in Art. 149 Abs. 2 lit. f. bestimmt, dass ausländische Entscheidungen über Ansprüche aus unerlaubter Handlung in der Schweiz nur dann anzuerkennen sind, wenn der Beklagte u.a. seinen Wohnsitz nicht in der Schweiz hatte, ermöglicht das LugÜ in Art. 5 Ziff. 3 dem Patienten, eine unerlaubte Handlung am Gericht des Ortes einzuklagen, an dem das schädigende Ereignis eingetreten ist. Bei diesem Ort wird es sich im Falle einer Beratung von im Ausland lebenden Personen mit Körperschaden um den ausländischen Wohnsitz des betreffenden Patienten handeln. Wie bereits erwähnt, würde ein solcher Entscheid hier in der Schweiz anerkannt und vollstreckt (Art. 25 und 31 LugÜ).

\section{Gerichtsstandsvereinbarung und Vereinbarung des anwendbaren Rechts}

Grundsätzlich besteht für die Vertragsparteien die Möglichkeit, sowohl bezüglich des Gerichtsstandes als auch bezüglich des anwendbaren Rechts eine Rechtswahl zu treffen und beispielsweise für allfällige Streitigkeiten das Gericht am Wohnsitz einer Vertragspartei zuständig und schweizerisches Recht als anwendbar zu erklären. Eine solche Erklärung hat jedoch nur Gültigkeit, wenn sie zwischen den Parteien ausdrücklich vereinbart worden ist. Wieweit eine entsprechende Erklärung des Arztes auf der Website oder auf dem vom Patienten auszufüllenden Fragenformular im Streitfall von den Gerichten als rechtsgenügende Vereinbarung beurteilt würde, wird die Gerichtspraxis weisen.

An dieser Stelle muss aber sogleich darauf hingewiesen werden, dass im internationalen Verhältnis in gewissen Fällen, so z. B. im Zusammenhang mit Konsumentenverträgen/Verbrauchersachen, von Gesetzes wegen die Vereinbarung eines Gerichtsstandes oder eines bestimmten anzuwendenden Rechts ausgeschlossen ist [28], so dass eine von den Parteien getroffene Wahl für einen inländischen Gerichtsstand und für die Anwendung von schweizerischem Recht unter Umständen als ungültig bezeichnet werden müsste. Dasselbe gilt auch im Binnenverhältnis: Der Konsument kann gegenüber dem Vertragspartner nicht zum voraus gültig auf den Gerichtsstand an seinem Wohnsitz verzichten [29].

Allerdings ist dem Autor noch kein höchstrichterlicher Entscheid bekannt, wonach das Arzt-Patienten-Verhältnis ausdrücklich als Konsumentenvertrag im Sinne des schweizerischen Gerichtsstandsgesetzes bezeichnet wurde.

Angesichts der aufgezeigten Unbestimmtheiten ist jedem Arzt zu empfehlen, auf seiner Website oder auf dem Fragenformular, das vom Patienten ausgefüllt werden muss, die Erklärung anzubringen, dass das Vertragsverhältnis dem Schweizer Recht untersteht und als Gerichtsstand die Gerichte am Wohnsitz des Arztes zuständig sind. Dies getreu nach dem Motto: «Nützt's nichts, schadet's nichts.» 


\section{Fazit}

Das Ausgeführte lässt sich wie folgt zusammenfassen und ergänzen:

- Die Beratung von Patienten via Internet kann einem echten Bedürfnis entsprechen und verstösst nach Ansicht des Autors nicht gegen die Standesregeln der FMH. Allerdings ist im Einzelfall sorgsam abzuwägen, ob eine seriöse Antwort ohne Konsultation des Patienten überhaupt möglich ist. Im Zweifelsfall ist der Patient aufzufordern, vorstellig zu werden, einen andern Arzt seines Vertrauens aufzusuchen oder sich an einen (andern) Spezialisten zu wenden.

- Bei der Beratung via Internet ist als Ort der Berufsausübung der Wohnort des angefragten Arztes bzw. der Ort seiner Praxis zu betrachten. Bei dieser Form der Beratung handelt es sich nicht um eine ausserkantonale Berufsausübung oder um eine solche im Ausland. Das Einholen einer entsprechenden Bewilligung oder die Pflicht zur Registrierung entfällt.

- Bei der Online-Beratung ist von einem Vertragsverhältnis zwischen Arzt und Patient auszugehen, unabhängig davon, ob die Beratung unentgeltlich erfolgt oder gegen Bezahlung.

- Dem Umstand der Unentgeltlichkeit der Beratungstätigkeit kann und sollte der Richter, der schweizerisches Recht anwendet und einen allfälligen Kunstfehler der Arztes bejaht, bei der Schadenersatzbemessung Rechnung tragen und die Schadenersatzpflicht des Arztes billigerweise herabsetzen.

- Bei der Beratung von Personen im Ausland ist Vorsicht geboten: Es besteht die Gefahr, dass ein ausländisches Gericht auf die Klage gegen den Arzt eintritt und das ergangene Urteil in der Schweiz anerkannt und vollstreckt wird. Diese Gefahr besteht insbesondere dann, wenn ein Gericht das Vorliegen einer Verbrauchersache (Konsumentenvertrag) bejaht oder das Handeln des Arztes nach den Bestimmungen über die unerlaubte Handlung beurteilt.

- Der ausdrückliche Hinweis an den Patienten, dass im Streitfall schweizerisches Recht vor dem Gericht am Wohnsitz des Arztes zur Anwendung gelangen soll, gibt dem Arzt keine Gewähr, dass er nicht doch im Ausland nach dem dort geltenden Recht verklagt werden kann und ein ausländisches Urteil hier in der Schweiz anerkannt und vollstreckt würde.

- Der Arzt ist aufgrund der nur schwer abschätzbaren Risiken bei der Online-Behandlung von Personen im Ausland gut beraten, wenn er davon nur zurückhaltend Gebrauch macht oder, um allfälligen Konsumentengerichtsständen oder deliktsrechtlichen Gerichtsständen im Ausland zu entgehen, beispielsweise bereits auf seiner Website mit einer entsprechenden Erklärung darauf hinweist, dass er Anfragen von Personen aus bestimmten Ländern nicht beantworten werde.

\section{Literatur}

1 CASH, 19.11.1999.

2 http://www.usz.ch oder

https://www.rehabnet.ch:443/usz-hotline/.

3 http://www.marktplatz-tv.ch/merkblatt/ merkblatt 16042000.html.

4 Arztbesuch per Anruf oder per Mausklick? NZZ, Nr. 34, 10./11. Februar 2001. S. 15.

5 Iff HW, Kuhn HP. Telemedizin und Standesrecht. Schweiz Ärztezeitung 1999;80(35):2126-30 oder http://www.fmh.ch/d/43/obj_843/Telemedizin.htm.

6 Standesordnung FMH (12. Dezember 1996): http://www.fmh.ch/fmh.cfm? $\mathrm{I}=\mathrm{dCta}=4$.

7 Systematische Sammlung des Bundesrechts: SR 811.11.

8 Systematische Sammlung des Bundesrechts: SR 943.02.

9 Gesetzessammlung des Kantons Zürich: LS 810.1.

10 http://www.publex.de/coi-bin/prt.coi/Rechtsquellen/ Gesetze/Europa/rl200031eg.html.

11 Hanika H. Telemedizin - Handlungs- und Weiterentwicklungsbedarf. Med R 2001;3:110.

12 Entscheid des Schweizerischen Bundesgerichts vom 23. Oktober 1990: BGE 116 II 523.

13 Kuhn M. Die rechtliche Beziehung zwischen Arzt und Patient. In: Honsell H. (Hrsg.). Handbuch des Arztrechts. Zürich: Schulthess; 1994. S. 21.

14 Entscheid des Schweizerischen Bundesgerichts vom 3. November 1987: BGE 113 II 432.

15 Art. 6 OR.

16 Art. 395 OR.

17 Entscheid des Schweizerischen Bundesgerichts vom 20. September 1990: BGE 116 II 699.

18 Systematische Sammlung des Bundesrechts: SR 272.

19 Fellmann W. Berner Kommentar. Bern: Stämpfli; 1992. N 508 zu Art. 398 OR.

20 Systematische Sammlung des Bundesrechts: SR 291.

21 Keller M, Kren Kostkiewicz J. In: Heini A, Keller M, Siehr K. Vischer F, Volken P (Hrsg.). IPRG-Kommentar. Zürich: Schulthess; 1993. N 17 zu Art. 120 IPRG.

22 Systematische Sammlung des Bundesrechts: SR 0.275.11.

23 IPRG. Art. 120 Abs. 1 lit. b.

24 Rosenthal D. Projekt Internet. Zürich: Finanz und Wirtschaft; 1997. S. 345.

25 Arter 0, Jörg F, Gnos U. Zuständigkeit und anwendbares Recht bei internationalen Rechtsgeschäften mittels Internet unter Berücksichtigung unerlaubter Handlungen. AJP 2000;3:289.

26 Stäheli T. Kollisionsrecht auf dem Information Highway. In: Hilty RM (Hrsg.). Information Highway. Bern: Stämpfli; 1996. S. 617.

27 Weber-Stecher UM. Internationales Konsumvertragsrecht. Zürich: Schulthess; 1997. S. 59.

28 Art. 114 Abs. 2 und Art. 120 Abs. 2 IPRG sowie Art. 15 LugÜ.

29 Art. 21 Abs. 1 lit. a und Art. 22 Abs. 1 lit. a des am 1. Januar 2001 in Kraft getretenen Gerichtsstandsgesetzes (GestG). 